\title{
Elevated expression of AGGF1 predicts poor prognosis and promotes the metastasis of colorectal cancer
}

\author{
Xin Zhang ${ }^{1 \dagger}$, Huimin Sun ${ }^{2 \dagger}$, Wanyuan Chen ${ }^{1 *}$ and Xianglei He ${ }^{1 *}$ (D)
}

\begin{abstract}
Background: Angiogenic factor with G-patch and FHA domains 1 (AGGF1) can promote angiogenesis and increasing evidence has highlighted the important roles of AGGF1 in tumorigenesis. However, the differential expression as well as the biological functions of AGGF1 in colorectal cancer (CRC) remain to be established. The purpose of the present study is therefore to identify the effect of AGGF1 on prognosis and metastasis in CRC patients.
\end{abstract}

Methods: The expression level of AGGF1 in CRC was examined by qPCR, western blot and immunohistochemistry in a tissue microarray containing 236 CRC specimens and paired normal mucosae. And the effect of AGGF1 on CRC cell malignance was investigated in our established stable AGGF1 upregulated and knockdown CRC cell lines.

Results: The expression level of AGGF1 in CRC tissue was not significantly different to that in adjacent normal mucosa at the mRNA level. However, at the protein level, AGGF1 expression in CRC tissues was significantly higher than in paired normal mucosa, which showed a clear association with TNM stage, AJCC stage, vascular invasion, and differentiation. Further, we revealed an apparent correlation between AGGF1 expression and poorer disease-free survival and overall survival of CRC patients. In addition, we discovered that AGGF1 significantly promoted CRC cell wound healing, migration, and invasion in vitro and distant metastasis in vivo.

Conclusions: Our study demonstrates the aberrant overexpression of AGGF1 in CRC and provides a basis on which to explore the application of AGGF1 as a potential therapeutic target for CRC patients, especially for CRC patients with distant metastasis.

Keywords: Colorectal cancer, AGGF1, Prognosis, Metastasis

\section{Background}

Colorectal cancer $(\mathrm{CRC})$ is the third most commonly diagnosed cancer in males and the second in females around the world [1]. Attributed to CRC screening and/or a variety of therapies for advanced metastatic CRC being available, the mortality rates of CRC have decreased in recent years $[1,2]$. However, outcomes remain suboptimal due to a lack of efficient targets for postponing, even preventing the invasion and metastasis of CRC [3]. Accordingly, discovering targets involved in CRC progression and

\footnotetext{
* Correspondence: chenwanyuan@hmc.edu.cn; hexiangleizj@163.com

${ }^{+}$Xin Zhang and Huimin Sun contributed equally to this work.

'Department of pathology, Zhejiang Provincial Peoples' Hospital, Peoples'

Hospital of Hangzhou Medical College, Hangzhou 310014, China

Full list of author information is available at the end of the article
}

elucidating the mechanism by which they induce metastasis appears to be particularly urgent.

Over the past decade, our understanding of vascular endothelial growth factor (VEGF), which is essential for both physiological and pathological angiogenesis, has increased at an explosive rate, leading to the approval of anti-angiogenic drugs for cancer $[4,5]$. The angiogenic factor with G-patch and FHA domains 1 (AGGF1) protein is released outside endothelial cells when angiogenesis starts, promoting angiogenesis as strongly as VEGF $[6,7]$. The full-length AGGF1 complementary DNA (4049 base pairs) contains a long open reading frame and encodes a protein of 714 amino acids [8, 9]. AGGF1 ubiquitously expresses in human normal cells as well as cancer cells [6]. Numerous studies have reported the

(c) The Author(s). 2019 Open Access This article is distributed under the terms of the Creative Commons Attribution 4.0 International License (http://creativecommons.org/licenses/by/4.0/), which permits unrestricted use, distribution, and 
aberrant expression of AGGF1 in cancer tissues and its involvement in malignant progression, such as in gastric cancer, hepatocellular carcinoma, and serous ovarian cancer [10-12]. However, whether AGGF1 also promotes the progression of CRC and the underlying role of AGGF1 in CRC progression remains to be elucidated.

In the present study, we first identified the expression patterns of AGGF1 at both the mRNA and the protein level in CRC tissues. Then, by examining AGGF1 expression in a tissue microarray, we evaluated the association between its expression and clinicopathologic features of CRC patients. Further cell functional assays in vitro and in vivo were used to explore the roles of AGGF1 in CRC cell migration and invasion, aiming to reveal the promoting roles of AGGF1 in the development of CRC, especially in patients with distant metastasis.

\section{Methods}

\section{Patients and tissue specimens}

A total of 236 CRC patient samples were included at the time of diagnosis from patients treated at departments of general surgery, Zhejiang Provincial People's Hospital. Informed consent was obtained from all individual participants included in the study and the research was carried out in accordance with the World Medical Association Declaration of Helsinki. All patient-derived specimens were collected and archived under protocols approved by the institutional review boards of Zhejiang Provincial People's Hospital. Procedures were carried out in accordance with approved guidelines. Cancer tissues and their paired adjacent normal mucosa were collected immediately after surgical resection, then frozen in liquid nitrogen for subsequent RNA extraction or formalin-fixed and paraffin-embedded for immunohistochemical staining.

\section{Quantitative PCR (qPCR)}

The qPCR assays were performed as described previously [13-15]. The primers used for qPCR were: AGGF1, sense 5'-GGA CTA GTT TGC GAA AAC ATG GGT AGT G$3^{\prime}$ and antisense $5^{\prime}$-CCC AAG CTT TGC CTG CAA TGG TCT TTA TC-3'; GAPDH, sense 5'-GGG AAG GTG AAG GTC GGA GT-3' and antisense 5'-GGG GTC ATT GAT GCRC AAC A-3'.

\section{Western blot (WB) analysis}

The WB assays were performed as described previously [13-15]. Primary antibodies specific to AGGF1 (1:500, \# ab203680) and GAPDH (1:1000, \# ab8245) were purchased from Abcam (Cambridge, UK).

\section{Tissue microarray (TMA) construction and} immunohistochemical (IHC) staining

TMA construction was undertaken as reported previously [15]. The expression of AGGF1 in the TMA was tested using standard IHC staining methods. The staining intensity for AGGF1 was scored as follows: 0 (negative), 1 (weak), 2 (moderate), 3 (strong); and staining extent, based on the percentage of positively stained cells, was scored as follows: 0 (0\%), 1 (1-25\%), 2 (26-50\%), 3 (51-75\%), 4 $(76-100 \%)$. The sum of intensity and extent score were used as the final staining score: $0-2$, negative expression; $3-4$, weak expression; and 5-7, strong expression [16, 17]. The corresponding primary antibodies were: AGGF1 (1: 100, Abcam, Cambridge, UK).

\section{Cell culture and transfection}

Human CRC cell lines (SW480, SW620, HT-29, RKO, HCT-8, HCT-116, and LoVo) and a normal colon epithelial cell line (FHC) were purchased from the American Type Culture Collection (Manassas, VA, USA) in October 2018. All cell lines were cultured in Dulbecco's modified Eagle's medium (Gibco BRL, Grand Island, USA) supplemented with $10 \%$ fetal bovine serum, under a humidified atmosphere containing $5 \% \mathrm{CO}_{2}$ at $37^{\circ} \mathrm{C}$. Cell morphology was authenticated via microscope (Fluorescence Microscope Axiovert 200, Zeiss, Germany).

For gene expression manipulation studies, both small interfering RNA (siRNA) specially targeting AGGF1 and pcDNA3.1-AGGF1 plasmid and their control sequences were obtained from Obio Technology Co., Ltd. (Shanghai, China). CRC cells were transfected using Lipofectamine 2000 following the manufacturer's instructions.

\section{Colony formation assays}

Colony formation assays were used to evaluate the colony formation abilities of CRC cells and colony formation was determined by preparing single cell suspension solutions and seeding in 6-well plate with 800 cells/well. After a 14-day incubation, the cell colonies were fixed in methyl alcohol for $15 \mathrm{~min}$ and dyed with crystal violet for $15 \mathrm{~min}$. Colonies were then counted, and the plates were photographed.

\section{Wound healing assays}

Transfected cells were trypsinized and seeded into 6well plates $\left(1.0 \times 10^{5}\right.$ cells/well $)$. Upon reaching the exponential growth phase, cells were wounded with a sterile pipette tip and then washed with PBS. Images were captured at $0,12,24$, and $36 \mathrm{~h}$ intervals, and wound widths were quantified and compared with baseline values. Experiments were carried out in triplicate. 


\section{Transwell assays}

The transwell 24-well Boyden chamber (Corning, USA) with an $8.0 \mu \mathrm{m}$ pore size polycarbonate membrane was used for the cell migration (without Matrigel) and invasion (with Matrigel) assays according to the manufacturer's protocol. Briefly, each group of cells $\left(5 \times 10^{4} /\right.$ chamber $)$ was plated in the upper chambers in $200 \mu \mathrm{l}$ serum-free media for $36 \mathrm{~h}$, while the bottom chambers contained $600 \mu \mathrm{l}$ media supplemented with 10\% FBS as a chemoattractant. Cells that migrated and invaded to the reverse side of chamber inserts were fixed by methyl alcohol and stained with $0.1 \%$ crystal violet.

\section{Animal assays}

To further investigate the metastatic effect of AGGF1 in vivo, we utilized a CRC metastasis model in fourweek-old male, specific pathogen-free BALB/C nude mice, which were purchased from Shanghai Research Center for Model Organisms and housed under pathogen-free conditions in the animal experiment center of Zhejiang Provincial People's Hospital. A total of $1 \times 10^{6}(200 \mu \mathrm{l})$ HCT-116/Lv-AGGF1 and RKO/siAGGF1 as well as their control cells were transfected with lenti-LUC virus and injected into the tail veins of grouped nude mice $(n=5)$, respectively. At weekly intervals, after ether inhalation, mice were subjected to i.p. injection with D-luciferin $(150 \mathrm{mg} / \mathrm{kg})$, then imaged $10 \mathrm{~min}$ later using the IVIS Illumina System (Caliper Life Sciences). After six weeks, the mice were euthanized by cervical dislocation. The lung and liver tissue samples were obtained, weighed, and embedded in paraffin. The in vivo assay using nude mice was approved by the Institutional Animal Care and Use Committee of Zhejiang Provincial People's Hospital. All animal protocols were approved by Zhejiang Provincial People's Hospital Animal Care.

\section{Statistical analysis}

The Student's t-test was used to determine the differences in AGGF1 mRNA expression between CRC tissues and adjacent normal mucosa. The statistical significance of differences between AGGF1 expression and clinicopathological variables was estimated by the $x^{2}$ test or Fisher's exact test. Survival curves were calculated by the Kaplan-Meier method with the log-rank test. The hazard ratio with a $95 \%$ confidence interval in Cox proportional hazards regressions were applied to estimate the hazard risk of individual factors for overall survival (OS) and disease-free survival (DFS). $p<0.05$ with a two-sided test was considered to be statistically significant. All statistical analyses were carried out using the SPSS 22.0 statistical software package (SPSS, Chicago, IL, USA).

\section{Results}

AGGF1 protein rather than mRNA showed aberrant expression in CRC

Using qPCR, we first detected the expression level of AGGF1 in 30 pairs of randomly selected CRC specimens. However, no clear difference was observed between CRC tissues and paired normal mucosa at the mRNA level (Additional file 1: Fig. S1). Consistent with this, upon further analysis of four public databases from Oncomine (https://www.oncomine.org/resource/main. html) we did not find significantly different AGGF1 mRNA expression between CRC tissues and normal mucosa (Additional file 2: Fig. S2). Subsequently, we examined the protein level of AGGF1 in eight samples randomly selected from the 30 paired specimens mentioned above by WB assay and found that the expression of AGGF1 protein was significantly upregulated in CRC tissues compared to paired normal mucosa (Fig. 1a). Taken together, these data suggest that aberrant expression of AGGF1 in CRC tumorigenesis and development may play significant roles at the protein level rather than at the mRNA level.

\section{Correlations between AGGF1 protein expression and clinicopathological features in CRC}

To clarify the correlations between AGGF1 protein expression and clinicopathological features of CRC patients, we first examined the expression of AGGF1 in a TMA by immunohistochemical (IHC) staining. As summarized in Additional file 6: Table S1, of the 236 normal specimens in the TMA, 129 (54.7\%) showed negative AGGF1 expression (Fig. 1b,f and control experiments were showed in Additional file 3: Fig. S3), 68 (28.8\%) had weak positive staining, and 39 (16.5\%) displayed strong positive staining. In contrast, the immunoreactive patterns of AGGF1 were predominantly positive in the majority of CRC specimens, with 76 (32.2\%) cases showing weak positive staining and 114 (48.3\%) exhibiting strong positive staining (Fig. 1c-e and g-i). These results further confirm that the expression levels of AGGF1 in CRC tissues were higher than those in adjacent normal mucosa $(p<0.001)$.

The correlation between AGGF1 expression and clinicopathologic features of CRC patients is summarized in Table 1. Aberrant upregulated expression of AGGF1 was highly correlated with tumor invasion $(p<0.001)$, LNM $(p<0.001)$, distant metastasis $(p=0.012)$, advanced AJCC stage $(p<0.001)$, and tumor recurrence $(p=0.004)$. On the other hand, no significant association was found between AGGF1 expression and age, gender, tumor location, or differentiation $(p>0.05$ for all, Table 1). All of these data further reveal the aberrant expression of AGGF1 in CRC tissues and its association with a more malignant CRC phenotype. 


AGGF1
AGGF1

\section{High expression of AGGF1 predicted poorer prognosis in CRC}

Using Kaplan-Meier curves with a log-rank test for disease-free survival (DFS) and overall survival (OS), we assessed the predictive role of AGGF1 in CRC patient survival and found that patients with AGGF1-positive tumors had poorer DFS and OS rates than those with AGGF1negative tumors ( $p<0.05$ for both, Fig. 2a-b). More interestingly, in CRC patients at AJCC stage III-IV, higher AGGF1 expression was significantly associated with poorer OS ( $p=0.001$, Fig. $2 \mathrm{~d})$, while no significant difference between higher and lower AGGF1 expression was observed for OS in patients at AJCC stage I- II, ( $p=$ 0.2248 , Fig. 2c). Additionally, univariate and multivariate survival analyses for DFS and OS were performed using the Cox proportional hazards model and the results demonstrated that AGGF1 expression $(p<0.05$, Table 2 and Table 3) could serve as an independent prognostic factor for DFS and OS in CRC patients. Interestingly, we also analyzed the impact of aberrant AGGF1 mRNA expression on CRC patient survival by using the public dataset from TCGA and discovered that there was no significant association between the expression of AGGF1 and OS (Additional file 4: Fig. S4), which further demonstrates that AGGF1 plays significant roles in tumorigenesis and the progression of CRC at the protein level rather than the mRNA level.

\section{AGGF1 promotes CRC cell migration and invasion in vitro} and distant metastasis in vivo

To further investigate the roles of AGGF1 on CRC progression, we first detected the expression of AGGF1 in seven CRC cell lines and one healthy colorectal cell line at the protein level. Then, according to the discrepant expression levels of AGGF1, we selected HCT-116 and RKO, which showed the lowest and highest expression level of AGGF1, respectively, to further investigate the effect of AGGF1 on CRC biological processes (Fig. 3a). Subsequently, by viral transfection, we generated a stable AGGF1-overexpressing HCT-116 cell line and an AGGF1-knockdown RKO cell line, with the ideal efficiency evaluated by WB (Fig. 3b).

Firstly, we performed cell colony formation assays to explore the underlying roles of AGGF1 on CRC cells' 
Table 1 Associations between AGGF1 expression and clinicopathological characteristics in 236 CRC patients

\begin{tabular}{|c|c|c|c|c|c|}
\hline \multirow[t]{2}{*}{ Variable } & \multirow[t]{2}{*}{$\mathrm{n}$} & \multicolumn{3}{|c|}{ AGGF1 expression } & \multirow[t]{2}{*}{$p$ value } \\
\hline & & Negative (46) & Weak positive (76) & Strong positive (114) & \\
\hline Age (yr) & & & & & 0.837 \\
\hline$<57$ & 112 & $22(19.6 \%)$ & $34(30.4 \%)$ & $56(50.0 \%)$ & \\
\hline$>=57$ & 124 & $24(19.4 \%)$ & $42(33.9 \%)$ & $58(46.8 \%)$ & \\
\hline Gender & & & & & 0.359 \\
\hline Male & 139 & $23(16.5 \%)$ & $45(32.4 \%)$ & $71(51.1 \%)$ & \\
\hline Female & 97 & $23(23.7 \%)$ & $31(32.0 \%)$ & $43(44.3 \%)$ & \\
\hline Tumor location & & & & & 0.381 \\
\hline Right & 49 & $5(10.2 \%)$ & $15(30.6 \%)$ & $29(59.2 \%)$ & \\
\hline Transverse & 5 & $1(20.0 \%)$ & $2(40.0 \%)$ & $2(40.0 \%)$ & \\
\hline Left & 12 & $2(16.7 \%)$ & $3(25.0 \%)$ & $7(58.3 \%)$ & \\
\hline Sigmoid & 49 & $7(14.3 \%)$ & $16(32.7 \%)$ & $26(53.1 \%)$ & \\
\hline Rectum & 121 & 31 (25.6\%) & $40(33.1 \%)$ & $50(41.3 \%)$ & \\
\hline T classification & & & & & $<0.001^{*}$ \\
\hline T 1 & 2 & $2(100.0 \%)$ & $0(0.0 \%)$ & $0(0.0 \%)$ & \\
\hline T2 & 36 & $21(58.3 \%)$ & 7 (19.4\%) & $8(22.2 \%)$ & \\
\hline T 3 & 44 & 7 (15.9\%) & $21(47.7 \%)$ & $16(36.4 \%)$ & \\
\hline T 4 & 154 & $16(10.4 \%)$ & $48(31.2 \%)$ & $90(58.4 \%)$ & \\
\hline N classification & & & & & $<0.001^{*}$ \\
\hline No & 108 & $36(33.3 \%)$ & $44(40.7 \%)$ & $28(25.9 \%)$ & \\
\hline N 1 & 77 & 7 (9.1\%) & $18(23.4 \%)$ & $52(67.5 \%)$ & \\
\hline N 2 & 51 & $3(5.9 \%)$ & $14(27.5 \%)$ & $34(66.7 \%)$ & \\
\hline M classification & & & & & $0.012^{*}$ \\
\hline MO & 204 & 44 (21.6\%) & $69(33.8 \%)$ & $91(44.6 \%)$ & \\
\hline M 1 & 32 & $2(6.3 \%)$ & 7 (21.9\%) & $23(71.9 \%)$ & \\
\hline AJCC stage & & & & & $<0.001^{*}$ \\
\hline I & 24 & 19 (79.2\%) & 5 (20.8\%) & $0(0.0 \%)$ & \\
\hline$\|$ & 76 & 15 (19.7\%) & $36(47.4 \%)$ & 25 (32.9\%) & \\
\hline III & 104 & 10 (9.6\%) & $28(26.9 \%)$ & $66(63.5 \%)$ & \\
\hline IV & 32 & $2(6.3 \%)$ & 7 (21.9\%) & 23 (71.9\%) & \\
\hline Recurrence & & & & & $0.004^{*}$ \\
\hline No & 199 & $43(21.6 \%)$ & $69(34.7 \%)$ & 87 (43.7\%) & \\
\hline Yes & 37 & $3(8.1 \%)$ & 7 (18.9\%) & 27 (73.0\%) & \\
\hline Differentiation & & & & & 0.640 \\
\hline Well & 50 & $8(16.0 \%)$ & 17 (34.0\%) & $25(50.0 \%)$ & \\
\hline Moderate & 173 & 34 (19.7\%) & 57 (32.92\%) & $82(47.4 \%)$ & \\
\hline Poor & 13 & $4(30.8 \%)$ & $2(15.4 \%)$ & 7 (53.8\%) & \\
\hline
\end{tabular}

*Significant difference

proliferation, and found that overexpression or knockdown of AGGF1 expression showed no significant promotion or inhibition on CRC cells' proliferation, respectively (Additional file 5: Fig. S5). Then, in vitro wound healing assays showed that ectopic overexpression or knockdown of AGGF1 could significantly accelerate or delay the wound healing ability of CRC cells, respectively, as compared with the control groups $(p<$ 0.05 for all, Fig. 3c). Transwell assays further demonstrated that upregulated or downregulated expression of AGGF1 could also strengthen or attenuate the migration and invasion ability, respectively, of CRC cells in vitro $(p<0.05$ for all, Fig. 3d). To investigate the functional impact of AGGF1 on distant metastasis of CRC in vivo, 

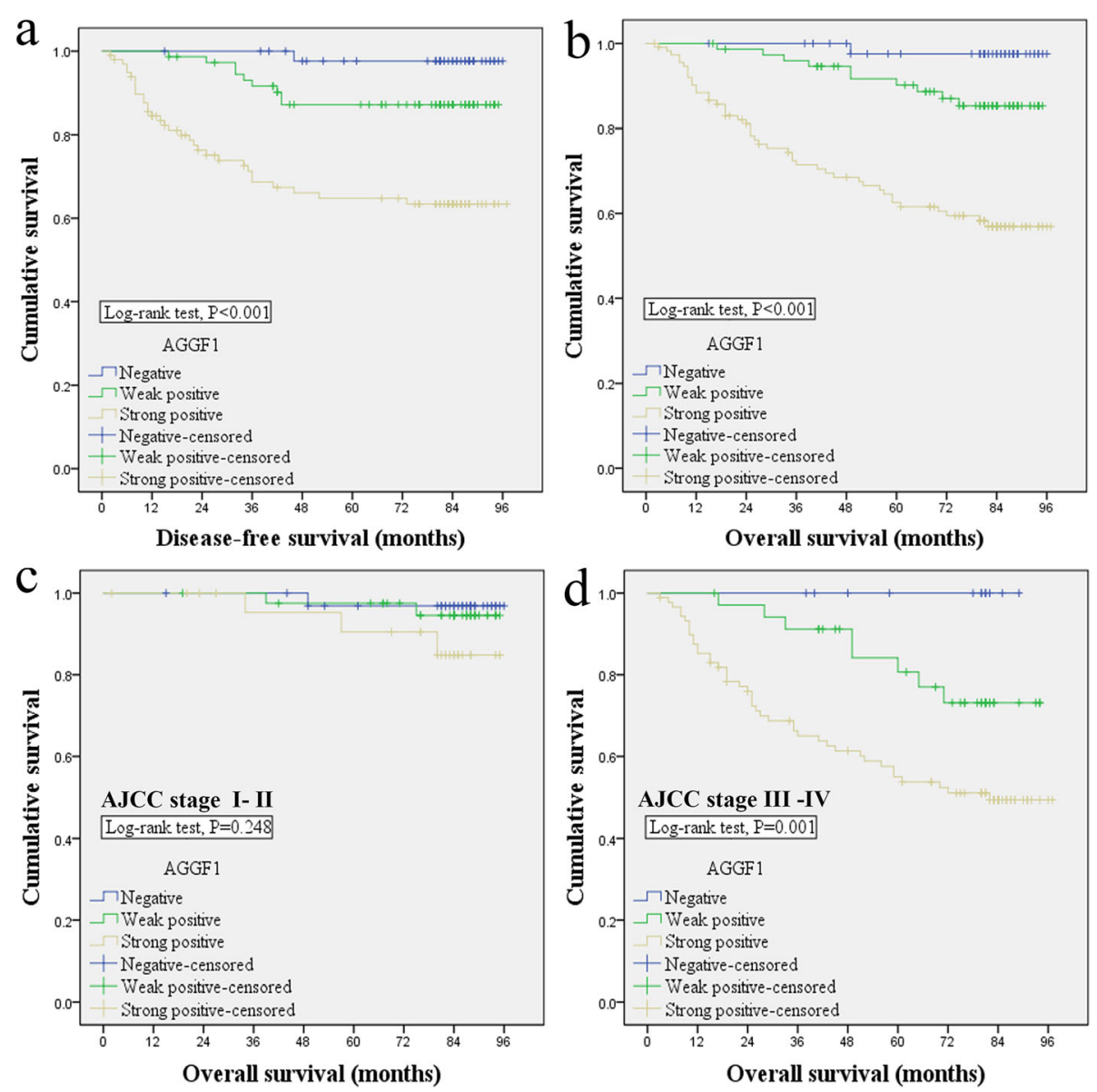

Fig. 2 Kaplan-Meier analysis with a log-rank test of survival. Disease-free survival (a) and overall survival (b) were significantly shorter in patients with AGGF1-positive tumors than in those with AGGF1-negative tumors ( ${ }^{*} p<0.05$ for both, log-rank test). P-values were calculated by log-rank test and $p<0.05$ was considered significant

Table 2 Univariate and multivariate analysis of disease-free survival in 236 CRC patients

\begin{tabular}{|c|c|c|c|c|}
\hline & \multicolumn{4}{|l|}{ Disease-free survival } \\
\hline & \multicolumn{2}{|l|}{ Univariate analysis } & \multicolumn{2}{|l|}{ Multivariate analysis } \\
\hline & $\mathrm{HR}(95 \% \mathrm{Cl})$ & $p$ Value & HR $(95 \% \mathrm{Cl})$ & $p$ Value \\
\hline Age (yr) & $0.871(0.476-1.595)$ & 0.655 & & \\
\hline Gender & $1.153(0.628-2.117)$ & 0.646 & & \\
\hline Tumor location & $0.997(0.816-1.220)$ & 0.980 & & \\
\hline T classification & $2.636(1.415-4.911)$ & $0.002^{*}$ & $2.773(1.238-6.211)$ & $0.013^{*}$ \\
\hline N classification & $2.514(1.480-3.136)$ & $<0.001^{*}$ & $0.744(0.415-1.334)$ & $0.007^{*}$ \\
\hline M classification & $6.733(3.281-13.815)$ & $<0.001^{*}$ & 9.195 (3.931-21.504) & $<0.001^{*}$ \\
\hline AJCC stage (III -IV vs I- II) & $6.160(2.593-14.635)$ & $<0.001^{*}$ & 3.968 (1.203-130.90) & $0.024^{*}$ \\
\hline Recurrence & $7.689(4.112-14.375)$ & $<0.001^{*}$ & $7.422(3.701-14.884)$ & $<0.001^{*}$ \\
\hline Differentiation & $0.666(0.368-1.206)$ & 0.180 & & \\
\hline AGGF1 & 3.956 (2.179-7.182) & $<0.001^{*}$ & $3.268(1.779-6.002)$ & $<0.001^{*}$ \\
\hline
\end{tabular}

HR: hazard ratio; Cl: confidence interval

* Significant differences 
Table 3 Univariate and multivariate analysis of overall survival in 236 CRC patients

\begin{tabular}{|c|c|c|c|c|}
\hline & \multicolumn{4}{|l|}{ Overall survival } \\
\hline & \multicolumn{2}{|l|}{ Univariate analysis } & \multicolumn{2}{|l|}{ Multivariate analysis } \\
\hline & $\mathrm{HR}(95 \% \mathrm{Cl})$ & $p$ Value & HR $(95 \% \mathrm{Cl})$ & $p$ Value \\
\hline Age (yr) & $0.895(0.530-1.512)$ & 0.679 & & \\
\hline Gender & $1.222(0.723-2.066)$ & 0.455 & & \\
\hline Tumor location & $1.168(1.000-1.364)$ & $0.050^{*}$ & $1.190(0.994-1.423)$ & 0.058 \\
\hline T classification & $3.182(1.705-5.937)$ & $<0.001^{*}$ & $2.938(1.404-6.149)$ & $0.004^{*}$ \\
\hline N classification & $2.243(1.615-3.115)$ & $<0.001^{*}$ & $0.912(0.586-1.419)$ & $0.002^{*}$ \\
\hline M classification & $9.412(5.481-16.162)$ & $<0.001^{*}$ & $9.385(4.907-17.952)$ & $<0.001^{*}$ \\
\hline AJCC stage (III -IV vs I- II) & 7.927 (3.394-18.511) & $<0.001^{*}$ & 3.369 (1.147-9.895) & $0.027^{*}$ \\
\hline Recurrence & $3.730(2.144-6.488)$ & $<0.001^{*}$ & $5.667(2.882-11.140)$ & $<0.001^{*}$ \\
\hline Differentiation & $0.616(0.370-1.025)$ & 0.062 & & \\
\hline AGGF1 & $4.182(2.384-7.333)$ & $<0.001^{*}$ & $3.320(1.814-6.077)$ & $<0.001^{*}$ \\
\hline
\end{tabular}

HR: hazard ratio; Cl: confidence interval

* Significant differences

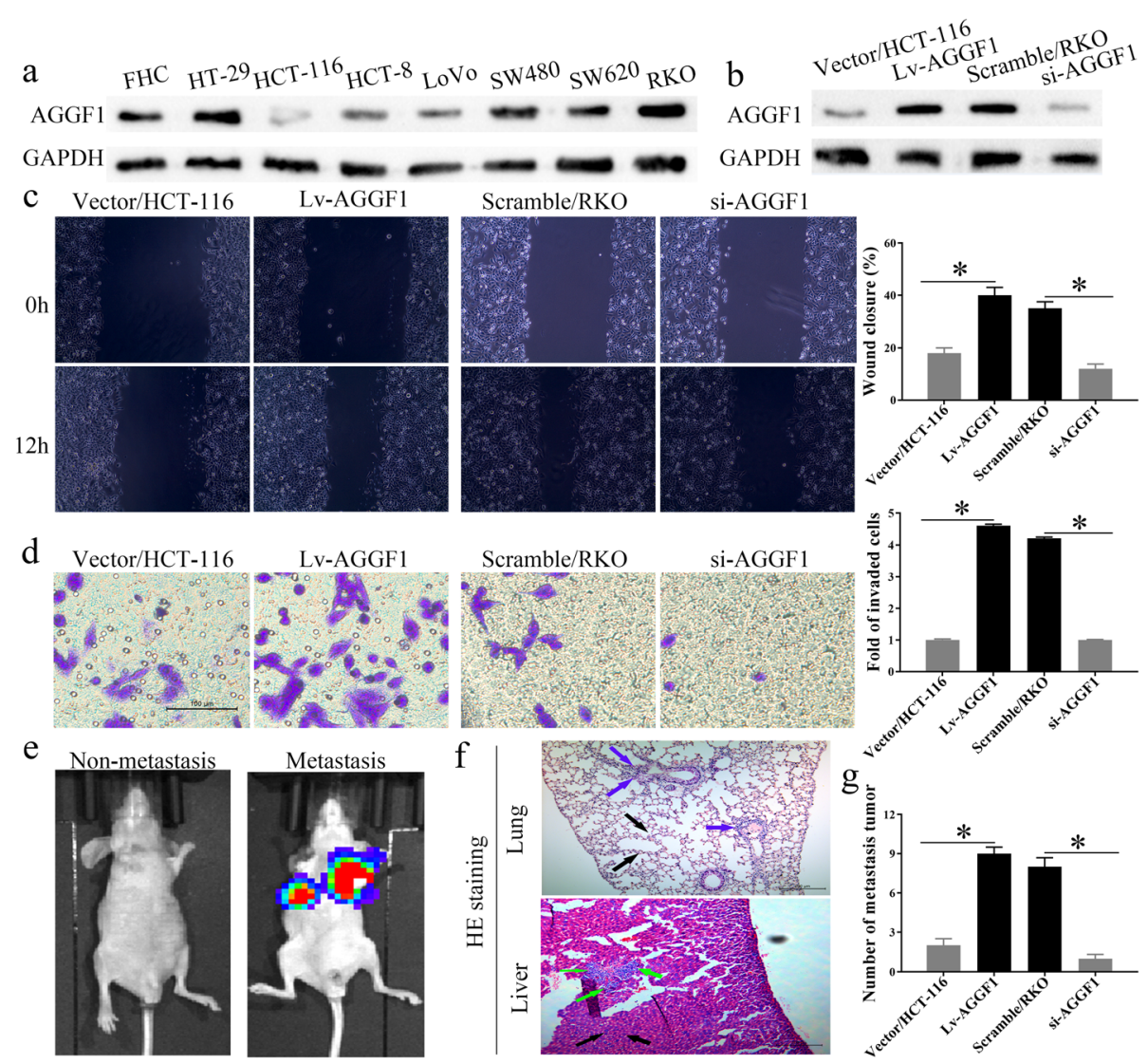

Fig. 3 In vitro and in vivo assays. a. he protein level of AGGF1 in one normal colorectal mucosa cell line (FHC) and seven CRC cell lines. The expression level of AGGF1 protein in HCT-116 and RKO cells transfected with AGGF1 overexpression or knockdown vectors was validated using western blotting (b). GAPDH was used to normalize protein level. In vitro, overexpression or knockdown of AGGF1 inhibited or elevated CRC wound healing (c), migration, and invasion (d), respectively, compared with control groups. Upregulation or knockdown of AGGF1 expression facilitated or suppressed the tumor metastasis ability of CRC cells in nude mice. e. Representative images of metastatic tumor colonies in mice (left panel (non-metastasis): the nude mouse without metastatic tumor colonies formed, right panel (metastasis): the nude mouse with metastatic tumor colonies formed). f. HE staining of the metastatic tumor colonies in lung and liver (colored arrows: metastatic tumor colonies). g. The number of metastatic tumor colonies was counted ( ${ }^{*} p<0.05$, c, f: Original magnification: $100 x$; d: Original magnification: 200x) 
in vivo fluorescence imaging of mice was performed to test the lung and liver metastasis of CRC (Fig. 3e). Then, hematoxylin-eosin (HE) staining was used to count the number of tumor colonies in mice lung and liver tissues (Fig. 3f). We found that AGGF1 overexpression or knockdown significantly aggrandized or weakened the formation of in vivo metastatic colonies compared with the control groups in both the lungs and livers of mice (Fig. 3g), $p<0.001$ ). Taken together, these data indicate that AGGF1 could facilitate the wound healing, migration, and invasion abilities of CRC cell in vitro and also promote distant metastasis in vivo.

\section{Discussion}

The prognosis for primary CRC is always favorable after curable surgical resection; however, the five-year OS rate of CRC patients with distant metastasis dramatically declines due to a lack of efficient drugs or treatment targets for postponing, even preventing the progression of CRC $[18,19]$. Thus, the identification of novel targets for CRC and further elucidation the mechanisms involved in the distant metastasis appears to be vitally important. In the present study, we demonstrated that the expression of AGGF1 was elevated in CRC tissues compared with corresponding normal mucosae, but only at the protein level. Aberrant overexpression of AGGF1 predicted poorer DFS and OS in CRC patients after radical surgery. Further, we verified that AGGF1 could accelerate CRC cell wound healing, migration, and invasion in vitro as well as distant metastasis in vivo. All of these findings support the hypothesis that ectopic expression of AGGF1 promotes CRC metastasis, serving as a potential therapeutic target for CRC patients, especially for patients with lung and liver metastasis.

Located on human chromosome 5q13.3, the AGGF1 mRNA gene encodes a protein consisting of 714 amino acids that shows strong expression in blood vessels and is secreted as vessel formation initiates [6, 20]. A series of studies have also reported that the expression level of AGGF1 in cancer tissues was clearly higher than in adjacent normal tissues, predicting poor prognosis [10-12]. In the present study, at the mRNA level, we found no difference in the AGGF1 expression between the CRC tissues and paired normal tissues, a result which was consistent with public databases from Oncomine. However, at the protein level, the expression level of AGGF1 in CRC tissues/cells was much higher than in corresponding normal mucosa/cells. Further results from IHC staining in a TMA revealed that positive AGGF1 protein expression was significantly correlated with poorer DFS and OS in CRC patients. However, public data from TCGA showed no obvious correlation between aberrant AGGF1 expression and OS, suggesting that AGGF1 is vitally importance in CRC malignant progression at the protein level rather than at the mRNA level.

As reported, AGGF1 is released outside endothelial cells when angiogenesis starts, promoting angiogenesis $[6,7]$, and increased angiogenesis is involved in the growth, metastasis, and survival of various tumors [21, 22]. Reduced expression of AGGF1 resulted in endothelial cell apoptosis and inhibition of endothelial capillary vessel formation and cell migration, which could be rescued by purified recombinant human AGGF1 protein [7]. To further elucidate the role of AGGF1 in CRC progression, we built the CRC cell lines HCT-116 and RKO with AGGF1 stable overexpression and knockdown, respectively. In vitro, we determined that AGGF1 promoted CRC cell wound healing, migration, and invasion, which indicates the potential involvement of AGGF1 in CRC metastasis and is consistent with the results of studies in gastric cancer [10] and hepatocellular carcinoma [11]. Additionally, results from the in vivo assay in nude mice showed that up- or downregulation of AGGF1 expression also led to a significant increase or reduction, respectively, in metastatic colonies formed in lungs and livers compared with the control groups. These in vivo and vitro data suggest that the elevated expression of AGGF1 is likely correlated with tumor invasion.

Tumor is known as a multi-gene/multi-step process. There is no doubt about the importance of TNMstage in tumor, however, even patients with the same TNM-stage may have different prognosis. Therefore, other molecules are still needed to supplement the TNM-stage to predict the prognosis and achieve accuracy study. For example, the immune scoring system can improve the assessment of the risk of CRC recurrence by introducing immune parameters for tumor staging [23]. The aberrant overexpression of AGGF1 in CRC can be detected by preoperative biopsy or postoperative immunohistochemistry, facilitating it served as a supplement to TNM staging. When patients with the same TNM-stage are accompanied by high expression of AGGF1, further intensive treatment should be specified to improve the prognosis of patients. Pre-operative radiotherapy or chemoradiotherapy is frequently used prior to CRC surgery to improve local control and survival [24]. The study on the clinical significance of AGGF1 in CRC patients with distant metastasis is conducive to the designation of personalized treatment strategy for CRC patients. Neo-adjuvant chemoradiotherapy could be adopted according to the patients' personal conditions, so as to achieve the degraded treatment of CRC and improve the postoperative outcomes of CRC patients. 


\section{Conclusions}

We demonstrated that AGGF1 expression was aberrantly elevated in CRC tissues and showed significant correlations with poor DFS and OS in CRC patients. In addition, we clarified that AGGF1 could promote CRC cell wound healing, migration, and invasion in vitro and distant metastasis in vivo, which indicates that AGGF1 may function as a potential therapeutic target for CRC patients, especially for patients with distant metastasis. Future studies will focus on the mechanisms underlying the role of AGGF1 in the progression of CRC and the potential for targeting AGGF1 in CRC treatment.

\section{Supplementary information}

Supplementary information accompanies this paper at https://doi.org/10. 1186/s12885-019-6474-7.

Additional file 1: Figure S1. The mRNA level of AGGF1 expression was detected in 30 pairs CRC specimens by qPCR. GAPDH was used as an internal control ( $\# p>0.05)$.

Additional file 2: Figure S2. Expression of AGGF1 at the mRNA level in four public databases from Oncomine. a. Ki Colon dataset, which is grouped as follows: no value $(n=41)$, colon squamous cell carcinoma ( $n=$ $3)$, colon adenocarcinoma $(n=77)$, and gastrointestinal stromal tumor $(n=$ 2). b. Graudens Colon dataset, which is grouped as follows: no value ( $n=$ 12) and colorectal carcinoma $(n=48)$. c. Gaspar Colon dataset, which is grouped as follows: no value $(n=22)$ and colorectal adenoma $(n=56) . d$ Sabates-Bellver Colon dataset, which is grouped as follows: no value ( $n=$ 32), rectal adenoma( $n=7)$, and colon adenoma $(n=25)$.

Additional file 3: Figure S3. Control experiments for antibody staining a. Original magnification: 50x; b. Original magnification: 400x

Additional file 4: Figure S4. Impact of AGGF1 expression at the RNA level on the OS of CRC patients in TCGA public databases. No significant correlation between aberrant expression of AGGF1 at the RNA level and OS was observed in CRC patients regardless of classification: a. Lower quartile classification; b. Lower tertile classification; c. Mean classification; d. Median classification; e. Upper quartile classification; f. Upper tertile classification

Additional file 5: Figure S5. The impact of AGGF1 on the proliferation of CRC cells was detected by cell colony formation assays in vitro. The results showed the impact of overexpression (a) as well as knockdown (b) of AGGF1 on CRC cells' growth, with the number of CRC cell clones were quantified (c).

Additional file 6: Table S1. Expression of AGGF1 in normal colorectal mucosa and primary cancerous tissues $(n=236)$.

\section{Abbreviations}

AGGF1: Angiogenic factor with G-patch and FHA domains 1; CRC: Colorectal cancer; DFS: Disease-free survival; IHC: Immunohistochemical; OS: Overall survival; TMA: Tissue microarray; VEGF: Vascular endothelial growth factor; WB: Western blot

\section{Acknowledgements}

Not applicable.

\section{Authors' contributions}

The study conception and design were made by XZ, HMS, WYC and XLH. Material preparation, data collection and analysis were performed by $X Z$ and HMS. The first draft of the manuscript was written by XZ and HMS, and all authors commented on previous versions of the manuscript. All authors read and approved the final manuscript.

\section{Funding}

This work was supported by Zhejiang Medical Technology Plan Project (grant number 2017ZD003). The funding bodies played no role in the design of the study or the collection, analysis, and interpretation of data or in writing the manuscript.

\section{Availability of data and materials}

The datasets used and analyzed during the current study are available from the corresponding author on reasonable request.

\section{Ethics approval and consent to participate}

Written informed consent was obtained from all individual participants included in the study and the research was carried out in accordance with the World Medical Association Declaration of Helsinki. All patient-derived specimens were collected and archived under protocols approved by the institutional review boards of Zhejiang Provincial People's Hospital. The in vivo assay using nude mice was approved by the Institutional Animal Care and Use Committee of Zhejiang Provincial People's Hospital. All animal protocols were approved by Zhejiang Provincial People's Hospital Animal Care.

Consent for publication

Not applicable.

\section{Competing interests}

The authors declare that they have no conflicts of interest

\section{Author details}

'Department of pathology, Zhejiang Provincial Peoples' Hospital, Peoples' Hospital of Hangzhou Medical College, Hangzhou 310014, China.

${ }^{2}$ Department of pathology, Weifang People's Hospital, Weifang 261041, China.

Received: 28 August 2019 Accepted: 17 December 2019

Published online: 27 December 2019

References

1. Torre LA, Bray F, Siegel RL, Ferlay J, Lortet-Tieulent J, Jemal A. Global cancer statistics, 2012. CA Cancer J Clin. 2015:65(2):87-108.

2. Siegel RL, Miller KD, Jemal A. Cancer statistics, 2015. CA Cancer J Clin. 2015; 65(1):5-29.

3. Sadeghi MR, Jeddi F, Soozangar N, Somi MH, Shirmohamadi M, Khaze V, et al. Nrf2/P-glycoprotein axis is associated with clinicopathological characteristics in colorectal cancer. Biomed Pharmacother. 2018;104:458-64.

4. Potente M, Gerhardt H, Carmeliet P. Basic and therapeutic aspects of angiogenesis. Cell. 2011;146(6):873-87.

5. Carmeliet P, Jain RK. Molecular mechanisms and clinical applications of angiogenesis. Nature. 2011:473(7347):298-307.

6. Tian XL, Kadaba R, You SA, Liu M, Timur AA, Yang L, et al. Identification of an angiogenic factor that when mutated causes susceptibility to KlippelTrenaunay syndrome. Nature. 2004;427(6975):640-5.

7. Fan C, Ouyang P, Timur AA, He P, You SA, Hu Y, et al. Novel roles of GATA1 in regulation of angiogenic factor AGGF1 and endothelial cell function. J Biol Chem. 2009;284(35):23331-43.

8. Durocher D, Henckel J, Fersht AR, Jackson SP. The FHA domain is a modular phosphopeptide recognition motif. Mol Cell. 1999;4(3):387-94.

9. Guglielmi B, Werner M. The yeast homolog of human PinX1 is involved in rRNA and small nucleolar RNA maturation, not in telomere elongation inhibition. J Biol Chem. 2002:277(38):35712-9.

10. Yao HH, Zhao YJ, He YF, Huang DB, Wang W. Knockdown of AGGF1 inhibits the invasion and migration of gastric cancer via epithelial-mesenchymal transition through Wnt/beta-catenin pathway. Cancer Cell Int. 2019;19:41.

11. Li W, Fu Q, Man W, Guo H, Yang P. LncRNA OR3A4 participates in the angiogenesis of hepatocellular carcinoma through modulating AGGF1/akt/ mTOR pathway. Eur J Pharmacol. 2019;849:106-14.

12. Zhao H, Sun Q, Li L, Zhou J, Zhang C, Hu T, et al. High expression levels of AGGF1 and MFAP4 predict primary platinum-based Chemoresistance and are associated with adverse prognosis in patients with serous ovarian Cancer. J Cancer. 2019;10(2):397-407.

13. Wang $X$, Luo G, Zhang $K$, Cao J, Huang C, Jiang T, et al. Hypoxic tumorderived Exosomal miR-301a mediates M2 macrophage polarization via 
PTEN/PI3Kgamma to promote pancreatic Cancer metastasis. Cancer Res. 2018;78(16):4586-98.

14. Mi Y, Zhao S, Zhang W, Zhang D, Weng J, Huang K, et al. Down-regulation of Barx2 predicts poor survival in colorectal cancer. Biochem Biophys Res Commun. 2016;478(1):67-73.

15. Mi Y, Zhao S, Zhou C, Weng J, Li J, Wang Z, et al. Downregulation of homeobox gene Barx2 increases gastric cancer proliferation and metastasis and predicts poor patient outcomes. Oncotarget. 2016;7(37):60593-608.

16. Zhang J, Liu L, Sun Y, Xiang J, Zhou D, Wang L, et al. MicroRNA-520g promotes epithelial ovarian cancer progression and chemoresistance via DAPK2 repression. Oncotarget. 2016;7(18):26516-34.

17. Zhao S, Jiang T, Tang H, Cui F, Liu C, Guo F, et al. Ubiquitin D is an independent prognostic marker for survival in stage IIB-IIC colon cancer patients treated with 5-fluoruracil-based adjuvant chemotherapy. J Gastroenterol Hepatol. 2015;30(4):680-8.

18. Liao F, He W, Jiang C, Yin C, Guo G, Chen X, et al. A high LDL-C to HDL-C ratio predicts poor prognosis for initially metastatic colorectal cancer patients with elevations in LDL-C. Onco Targets Ther. 2015;8:3135-42.

19. Notarnicola M, Lorusso D, Tutino V, De Nunzio V, De Leonardis G, Marangelli G, et al. Differential Tissue Fatty Acids Profiling between Colorectal Cancer Patients with and without Synchronous Metastasis. Int J Mol Sci. 2018;19(4): 962-9.

20. Timur AA, Driscoll DJ, Wang Q. Biomedicine and diseases: the KlippelTrenaunay syndrome, vascular anomalies and vascular morphogenesis. Cell Mol Life Sci. 2005;62(13):1434-47.

21. Xu Y, Zhou M, Wang J, Zhao Y, Li S, Zhou B, et al. Role of microRNA-27a in down-regulation of angiogenic factor AGGF1 under hypoxia associated with high-grade bladder urothelial carcinoma. Biochim Biophys Acta. 2014; 1842(5):712-25.

22. Folkman J, Shing Y. Angiogenesis. J Biol Chem. 1992;267(16):10931-4.

23. Pages F, Mlecnik B, Marliot F, Bindea G, Ou FS, Bifulco C, et al. International validation of the consensus Immunoscore for the classification of colon cancer: a prognostic and accuracy study. Lancet. 2018;391(10135):2128-39.

24. Hammarstrom K, Imam I, Korsavidou Hult N, Ekstrom J, Sjoblom T, Glimelius B. Determining the use of preoperative (chemo)radiotherapy in primary rectal cancer according to national and international guidelines. Radiother Oncol. 2019;136:106-12.

\section{Publisher's Note}

Springer Nature remains neutral with regard to jurisdictional claims in published maps and institutional affiliations.

Ready to submit your research? Choose BMC and benefit from:

- fast, convenient online submission

- thorough peer review by experienced researchers in your field

- rapid publication on acceptance

- support for research data, including large and complex data types

- gold Open Access which fosters wider collaboration and increased citations

- maximum visibility for your research: over $100 \mathrm{M}$ website views per year

At $\mathrm{BMC}$, research is always in progress.

Learn more biomedcentral.com/submissions 